\title{
MICROFLUIDIC DESIGN ENABLES LOW-INFRASTRCUTURE YET LARGE-SCALE SCREENING OF MOLECULAR BINDING REACTIONS \\ Yuchen Pan ${ }^{1}$, T.A. Duncombe ${ }^{1}$, C.A. Kellenberger ${ }^{2}$, M.C. Hammond ${ }^{2,3}$ and A.E. Herr ${ }^{*}$ \\ ${ }^{1}$ UC Berkeley / UC San Francisco Joint Graduate Group in Bioengineering, USA \\ ${ }^{2}$ Department of Chemistry, University of California, Berkeley, California, USA \\ ${ }^{3}$ Department of Molecular \& Cell Biology, University of California, Berkeley, California, USA
}

\section{ABSTRACT}

We detail the development and optimization of a low-infrastructure and high-throughput microfluidic electrophoretic mobility shift assays (EMSAs). The platform screens molecular binding in our recently published photopatterned free-standing polyacrylamide gel electrophoresis $(f s$ PAGE) system [1]. The new electrophoresis modality supports concurrent 96 separations and is operated with the standard equipment available in any experimental biology lab. We applied computational modeling of $f$ PAGE sample injection to minimize injection-dispersion and subsequent separation. In addition, a thermal model was established to analyze joule heating and gel evaporation, based on which two complimentary approaches (modified geometry and run buffer) were proposed to minimize the moisture loss and elongate the effective electrophoresis time course. Unit-to-unit variations in $f_{s}$ PAGE were quantified and used to inform design optimization which eliminates spatial variations across a 96-plex array. Lastly, a cyclic-di-GMP (CDG) riboswitch binding reaction was demonstrated in a 96-plex $f_{s}$ PAGE - showing good quantitative capability.

\section{INTRODUCTION}

As a crisis in antimicrobial resistance intensifies, novel antibiotics are urgently needed. Consequently, academic labs and federal agencies are ramping up efforts to tackle this health challenge. Molecular binding screening is one of the key tools in our arsenal to identify effective new compounds for the drug targets of interests. However, gold-standard screening tools (i.e., fluorescence polarization (FP) [2] \& Fluorescence resonance energy transfer (FRET) [3]) are sample-consuming, slow, and require substantial infrastructure. These functional limitations are exaggerated by scarce compound library resources, which lead to weak statistical validation. Even though droplets microfluidic has been introduced to improve the throughput of FP [4] and FRET [5], the complex technical facilities are way beyond the scope of a standard biology laboratory.

To facilitate the measurements, we propose electrophoretic mobility shift assays (EMSAs) to analyze molecular binding. However, traditional EMSAs rely on slab-gel electrophoresis, a decades-old, slow and high sample-consuming technique, thus inadequate for a large-scale molecular screening. Several studies have detailed the efforts in bringing microfluidic electrophoresis to speed up the analysis, including 384-plex radial microfluidic capillary electrophoresis (Emrich et al., 2002 [6]), multiplexed Gradient Elution Moving Boundary Electrophoresis (Ross et al., 2008 [7]) and microfluidic $\mu$ MSA (Karns et al., 2013 [8]). Nevertheless, these technologies have their own challenges to adoption, including complex electric interfacing and difficult operation.

To overcome these severe limitations, we introduce a new screening framework for quantitative molecular binding discovery that tests miniscule sample volumes rapidly with substantially reduced infrastructure needs. For the first time, we report a microfluidic 96-plex screening platform to support concurrent electrophoretic mobility shift assays. $f_{s} \mathrm{PAG}$ is a mm-sized and um-tall gel structure photopolymerized on a piece of surface-functionlized substrate $\left(\right.$ GelBond ${ }^{\mathbb{B}}$ ) [1]. The gel is simply comprised of a customizable sample reservoir and a separation lane. The entire prototyping cycle takes only $30 \mathrm{~min}$ to complete, including mask design, printing and $f_{s}$ PAG fabrication. Our previous results show that 96 concurrent protein separations can be completed in $10 \mathrm{~min}$. The multiplexed analysis made the $f_{s} \mathrm{PAG}$ an ideal platform for massive screening assay. For operation, the platform only requires a commercial electrophoresis power supply. Fig. 1 shows the architecture of a 96-plex $f$ sPAG layout.

In this work, we optimize the platform to screen for riboswitches. Riboswitches are structured bacterial mRNA molecules that are considered promising and largely unexplored drug targets [9]. Compound screening aims to identify effective riboswitch binders. Upon ligand binding, riboswitches compact (change size) and result in an electrophoretic mobility shift between bound and unbound forms which is measured by high-performance EMSAs.

Here we discuss the key factors that affect a successful EMSA and detail engineering approaches to optimize $f_{S}$ PAGE in the following aspects: 1) low-dispersion injection; 2) minimization of evaporation during electrophoresis - due to the high joule heating; 3 ) reduced "unit-to-unit" variation across a 96-plex array. The optimized $f_{s} \mathrm{PAG}$ EMSAs is used to perform 96-plex concurrent EMSAs to evaluate the binding reaction of a CDG riboswitch.

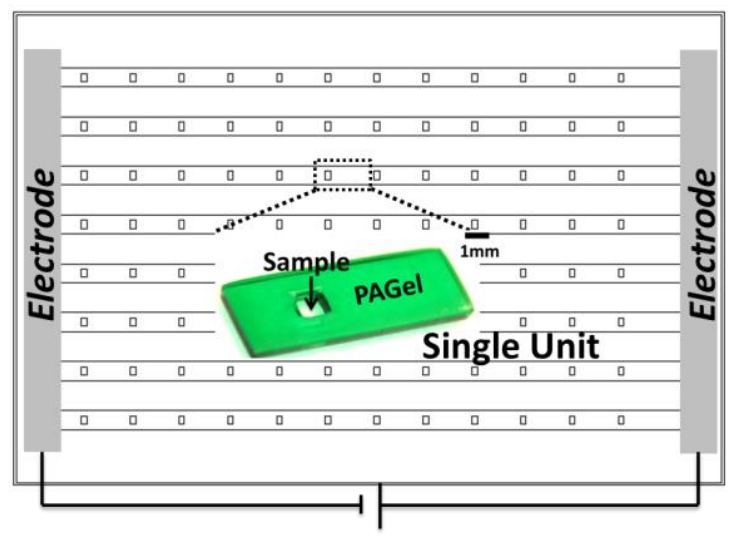

Figure 1: Layout of a 96-plex $f_{S} P A G$. The spacing of separation units correspond to a 96 well-plate format.

\section{MATERIALS AND METHODS}

\section{Materials}

Solutions of $30 \%(\mathrm{w} / \mathrm{v})(29: 1)$ acrylamide/bis-acrylamide, glycerol, glacial acetic acid and Triton X-100 were purchased from Sigma Aldrich (St. Louis, MO). 10X TBMK buffer was prepared in house by dissolving $890 \mathrm{mM}$ tris base (Fisher Scientific, Hampton, $\mathrm{NH}), 890 \mathrm{mM}$ boric acid (Fisher Scientific, Hampton, NH), $30 \mathrm{mM}$ magnesium chloride (EMD chemical, Gibbstown, NJ) and $100 \mathrm{mM}$ potassium chloride (Sigma Aldrich, St. Louis, MO) into $1 \mathrm{~L}$ water. Photoinitiator 2,2-azobis[2-methyl- N -(2-hydroxyethyl) propionamide] (VA-086) was purchased from Wako Chemical 
(Richmond, VA). GelBond ${ }^{\circledR}$ PAG film and Gel Slick ${ }^{\circledR}$ glass plate coating were purchased from Lonza (Base, Switzerland). AlexaFluor 488 (AF488) conjugated Trypsin Inhibitor (TI*, 21kDa), Ovalbumin (OVA*, 45kDa) and Transferrin (TRF*, 80kDa) were purchased from Life Technologies Corporation (Carlsbad, CA). Photo-masks were designed with AutoCad $^{\circledR}$ student edition (Autodesk, San Rafael, CA) and printed on a transparent film (3M, St. Paul, MN). Otherwise stated, all the reagents are prepared with molecular biology grade (DNase-, RNase- and Protease-free) water purchased from Mediatech (Manassas, VA).

Riboswitch RNAs were prepared using standard protocols. DNA templates were generated using primers that appended the T7 promoter sequence directly before the aptamer sequence. RNAs were then in vitro transcribed using T7 RNA polymerase following standard protocols. Following oxidation of the 3' ribose with $\mathrm{NaIO}_{4}$, AlexaFluor 488 was conjugated to the RNAs following standard procedures for 3' end labeling.

To prepare the binding reaction solution, riboswitch, 10X TBMK buffer, $1 \mathrm{mg} / \mathrm{ml}$ yeast tRNA and water are added and mixed in a non-stick surface $0.5 \mathrm{ml}$ eppendorf tube at indicated concentrations. The final mixture solution contains $1 \mathrm{X}$ TBMK buffer and $100 \mathrm{ug} / \mathrm{ml}$ yeast tRNA. The mixture is then heated at $70^{\circ} \mathrm{C}$ for $3 \mathrm{~min}$ and cooled at room temperature for $10 \mathrm{~min}$ to renature the RNA. Subsequently, cyclic-di-GMP at indicated concentration and was added into the solution along with an internal standard (TI*). The sample mixture were placed in dark and equilibrated at room temperature for 1 hour. The reaction was quenched by adding $1.5 \mathrm{ul}$ of $50 \%$ glycerol into every $10 \mathrm{ul}$ reaction.

\section{fsPAG fabrication}

$f_{s} \mathrm{PAG}$ was fabricated with UV photopatterning. The PAG precursor solution contained $20 \% \mathrm{~T}$ acrylamide, $3.3 \% \mathrm{C}$ bis-acrylamide crosslinker and 0.2\% VA-086 photo-initiator $(\mathrm{w} / \mathrm{v})$. The precursor solution was then degassed for 2-3min under house vacuum system with a sonicator and then subject to UV exposure. The choice of exposure time and intensity depends on the total acrylamide concentrations. For $20 \% f_{S} \mathrm{PAG}$, it takes 65 seconds under $20 \mathrm{~mW} / \mathrm{cm}^{2} \mathrm{UV}$.

\section{fsPAG electrophoresis operation}

Before electrophoresis, the fsPAG was soaked in the run buffer for $10 \mathrm{~min}$. Next, the fsPAG was taken out from the run buffer solution and placed into a custom designed environmental chamber [1] on top of a borosilicate glass. Electrode wicks (Serva, Heidelber, Germany) soaked in run buffer were aligned on two ends of a separation lane on top of the gel. Excess run buffer on the electrode wicks were removed with Kimwipe. Two graphite electrodes are placed right above in contact with the electrode wicks and were connected to the external power supplier. Sample solution was pipetted into the sample well. Eventually, the environmental chamber was sealed with a borosilicate glass plate. A voltage was applied using a PowerPac HV power supplier (Bio-Rad Laboratories) to initiate the electrophoresis. The power supplier enables real-time current reading. In the current monitoring experiments, the current was recorded manually every 10 seconds for up to $6 \mathrm{~min}$.

\section{fsPAG height measurement}

The height of the fsPAG was measured with MicroXAM-100 Optical Profilometer (ADE Phase Shift, Tucson, AZ).

\section{Imaging and data analysis}

Fluorescence imaging was conducted on an inverted epifluorescence microscope (Olympus IX-70) equipped with a $2 \mathrm{X}$ objective (PlanApo, N.A. $=0.08$, Olympus, Center Valley, PA) and an X-Cite ${ }^{\circledR}$ exacte mercury lamp (Lumen Dynamics, Mississauga, Canada). The light was filtered through a XF100-3 filter (Omega Optical, Battleboro, VT). A Peltier cooled charge-coupled device (CCD) camera (CoolSNAP HQ2, Roper Scientific, Trenton, NJ) attached to the microscope was used to capture image. Large area image acquisition was performed with Metamorph software (Molecular device, Sunnyvale, CA).

Post-processing of the image was conducted in ImageJ (NIH, Bethesda, MD) and subsequence data analysis was performed with OriginPro 8.0 (OriginLab, Northampton, MA). The intensity profiles were plotted over the transverse direction of the fsPAG.

\section{RESULTS AND DISCUSSION}

Low dispersion and high resolution separations are the goals for $f_{s}$ PAG EMSAs. This is crucial especially for molecular binding pairs that exhibit small mobility shifts upon interaction, such as riboswitch RNA. The previously published open channel 3-D structure of the $f_{s} \mathrm{PAG}$ renders less consistency in sample loading in terms of the height and potentially can suffer from premature termination of electrophoresis owing to moisture loss. In this work we study the sources of inconsistency and develop an optimized $f_{s}$ PAGE with improved reliability.

\section{Sample loading height}

Unlike its microfluidic counterpart, where sample injection and subsequent separations are achieved using additional channels, the two are combined in one step for $f s$ PAGE. Consequently, the analyte band shape can be distorted due to a non-uniform electric field distribution. Electric field non-uniformity can occur from both a gel-free solution conductivity mismatch and a height mismatch between the $f_{s} \mathrm{PAG}$ and the loaded sample. As a key aspect of the electrokinetic property of the $f s$ PAGE, the electric field distribution determines the migration velocity and therefore the quality of the separation. Fig. 2 shows COMSOL $^{\circledR}$ simulations demonstrating the relationship between analyte band shape and sample height. The simulations show that low sample loading can result in reverse skewing of injected analyte bands as well as streaking behind the main band. Further, low sample loading $(<20 \%)$ corresponds to a reduction in migration distance - caused by a lower electric field, as most of the voltage drop occurs across the sample well. Altogether, low sample heights lead to worse separation resolution (SR), making small mobility shifts difficult to resolve. SR is described by:

$$
S R=D / 2\left(\sigma_{1}+\sigma_{2}\right)
$$

Where $\mathrm{D}$ is the distance between two bands, $\sigma_{1}$ and $\sigma_{2}$ are the standard deviation of the bands. $>50 \%$ relative sample loading height is suggested to ensure the quality of the separation.

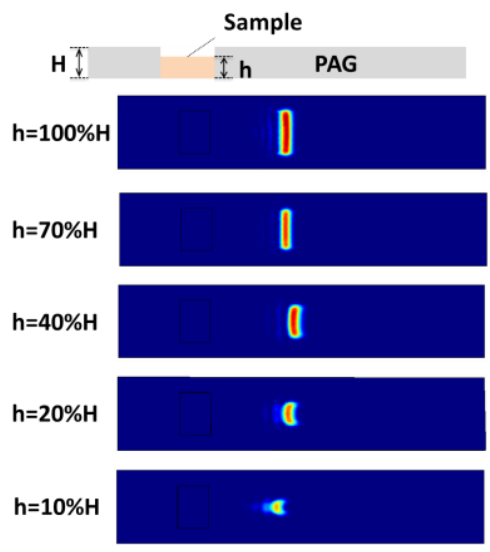


Figure 2: Comsol $^{\circledR}$ simulation results of $f_{S} P A G$ band shape for different sample loading height. Low sample loading ( $<20 \%)$ results in heavily skewed band and low migration distance, reducing the separation resolution.

\section{Thermal control of $f$ sPAG}

Evaporation is a problem for $f_{s} \mathrm{PAGE}$ due to its open nature. While microfluidic in-channel electrophoresis is generally operated under high electric field, the "sub-mm size" of the open $f_{s}$ PAG places limitation on the highest working voltage due to a worse heat dissipation that accelerates the evaporation and reduces the overall effective electrophoresis time. Theoretical analysis predicts that a lower temperature slows down water loss, and this can be accomplished by altering the surface area-volume ratio of the $f_{s} \mathrm{PAG}$ structures. In addition, glycerol was added into the run buffer to further slow evaporation. Fig 3A shows the current monitoring during $f_{s} \mathrm{PAG}$ electrophoresis up to $6 \mathrm{~min}$ under $60 \mathrm{~V} / \mathrm{cm}$ at different gel heights (run buffer contains $20 \%$ glycerol). We clearly observe improved stability in the relative electric current as $f s$ PAG height is reduced from $600 \mu \mathrm{m}$ to $100 \mu \mathrm{m}$, while no remarkable difference is observed between $70 \mu \mathrm{m}$ and $100 \mu \mathrm{m}$ devices. $f_{s}$ PAG smaller than $70 \mu \mathrm{m}$ is unreliable in both fabrication and in sample loading (due to sub 200nL volumes). In previously conducted in-chip CDG riboswitch separations (not shown), a 3min electrophoretic separation was required to fully resolve the CDG mobility shift. In Fig. 3B, we plotted the relative current drop at $3 \mathrm{~min}$ for each of the $f_{s}$ PAG heights tested. The $100 \mu \mathrm{m}$ structure was retained $91.3 \%$ of its initial current value, highest among all other gel heights.

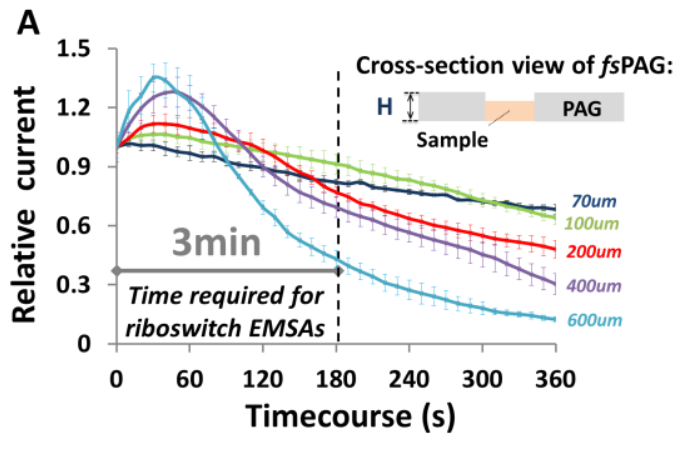

B

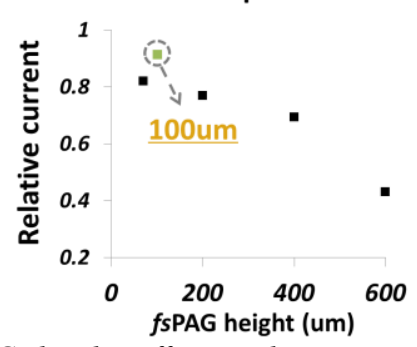

Figure 3: $f_{S} P A G$ height affects gel evaporation. A) Current monitoring of $f_{S} P A G$ electrophoresis for different gel heights up to 6 min. The current is normalized by the initial value for comparison. $E=60 \mathrm{~V} / \mathrm{cm}$. B) Current drop at 3min. Theoretical prediction suggests riboswitch binding pairs resolves at this time point.

Glycerol forms tight hydrogen bonds with water molecules and run buffer with glycerol experiences lower vapor pressure which slows down the evaporation process (data not shown here). In addition, glycerol adheres to the matrix of the gel and improves the structural integrity (data not shown here). One drawback of adding glycerol into run buffer is the increasing viscosity and therefore larger hydrodynamic resistance slows down the migration, which decreases the separation resolution. Therefore, a tradeoff between evaporation and migration velocity points to an optimal glycerol concentration that renders the highest separation quality. Experimental study on the relationship between the separation resolution and glycerol concentration indicates the optimal point is reached at $20 \%$ glycerol concentration.

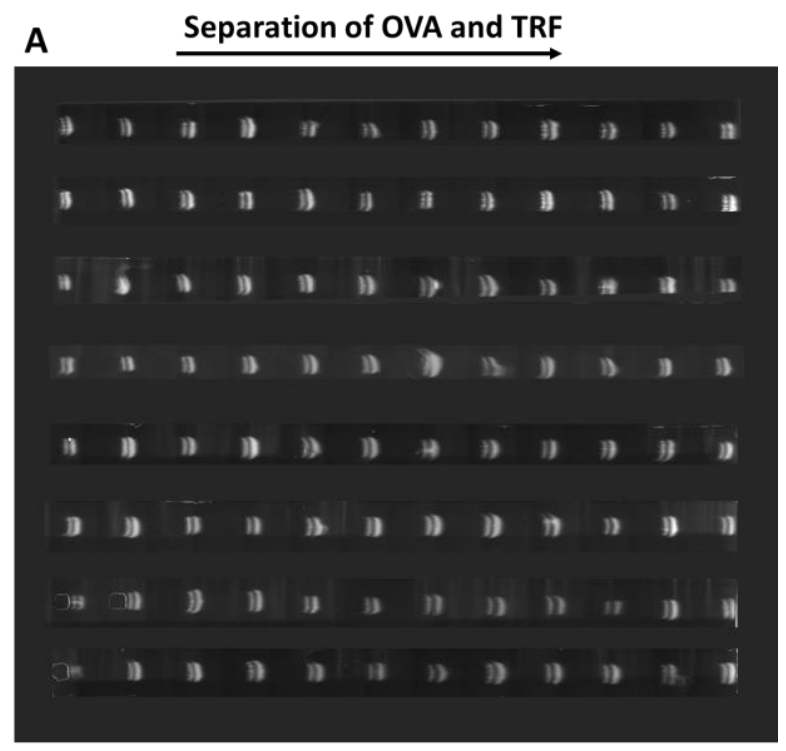

B

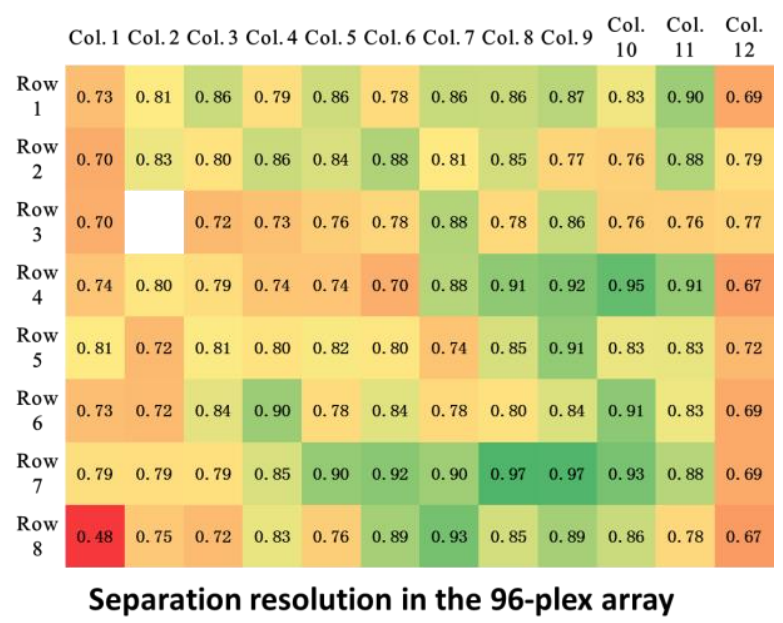

Figure 4: Electrophoresis of OVA and TRF on a 96-plex fsPAG platform. A) Fluorescence imaging of $O V A-T R F$ at 2 min. $E=60 \mathrm{~V} / \mathrm{cm}$. B) Heatmap of the $S R$ in each separation unit. The green cell has larger SR and red cell has smaller. Unit of row 3 and col 2 was not quantified due to a gel defects there

\section{Unit-to-unit variation}

Low unit-to-unit variation in analyte concentration, mobility and separation resolution are key aspects to developing a robust $f_{s} \mathrm{PAG}$ electrophoresis which facilitates parallel comparison between columns/lanes in the 96-plex separation and supports easier data analysis. Factors that can induce variations are: sample loading inconsistency (well shape and loading amount), mobility and electric field non-uniform distribution. Among these factors, loading inconsistency induces random errors and can be minimized with well-trained experimental operation or with automated fluid delivery. The mobility/electric field non-uniformity introduces 
systematic error and is studied here. We performed electrophoresis of Ovalbumin (OVA) and Transferrin (TRF) in a 100um tall $20 \% \mathrm{~T}$ fsPAG with 1x TBMK (20\% glycerol) for $2 \mathrm{~min}$ (Fig. 4A). To quantify any non-uniformity, the separation resolutions (indicative of both mobility and band width) for each unit are listed below (Fig. 4B). A heat map was generated based on the values to help analysis. Unit of row 3 and col 2 was not quantified due to a gel defects there. It can be seen from the heat map that the two columns on the sides have lower SR. This observation was further confirmed by ANOVA test $(\alpha=0.05)$. In addition, no row-to-row variation was observed. We believe this variation was a result of electrode side effects. The conjunction of electrodes and electric wicks expand the current conducting areas, which then act to spread out the electric field lines, therefore lowering the electric field strength.

To reduce the side effect, a new $f_{s} \mathrm{PAG}$ that has electrodes farther from the separation units are designed. For the same experiment, no significant side effects are observed (data not shown).

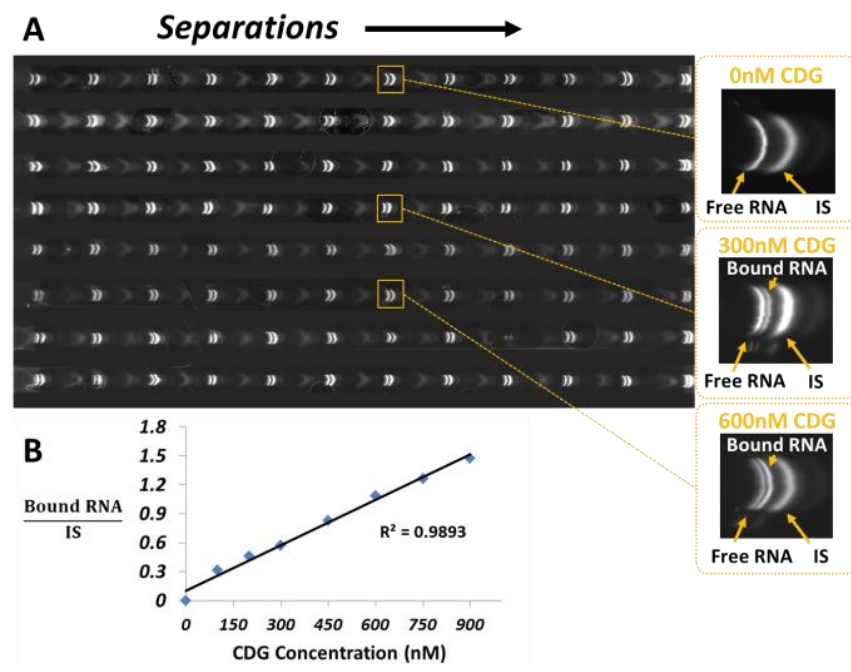

Figure 5: $C D G$ riboswitch EMSAs on a 96-plex $f_{S} P A G$. A) Fluorescence imaging of the separation at $3 \mathrm{~min}$. Three separation units at 0, 300, 600nM CDG were shown on the right. Increasing fluorescent signals of bound RNA are observed. IS: Internal Standard.

\section{Quantitative 96-plex fsPAG $\boldsymbol{\mu}$ EMSA of CDG riboswitch}

We investigated the binding reaction of CDG riboswitch on the $f s$ PAG as a first step towards a successful massive screening platform. Structural data has shown that the CDG riboswitch forms a more compact structure when in complex with its ligand, which indicates that the ligand-bound RNA should encounter less hydrodynamic resistance in the $f s$ PAG. The resultant larger electrophoretic mobility causes the ligand-bound RNA to separate from the ligand-free RNA in the gel matrix and the shift can be quantified to reveal the amount of CDG in the sample. The geometrical design for such a 96-plex ensures that the minimal distance in between the adjacent separation units should accommodate a full separation. As such, we devised a 96-plex $20 \% \mathrm{~T}$ fsPAG with $4.5 \mathrm{~mm}$ horizontal well-to-well spacing and $9 \mathrm{~mm}$ lane-to-lane distance. Fig. 5 shows the fluorescence imaging of the EMSAs results. The reactions were prepared by incubating $1 \mathrm{uM}$ riboswitch with increasing concentration of CDG (ranging from 0 $\mathrm{nM}$ to $900 \mathrm{nM}$ ). 500nM trypsin inhibitor is used as internal standard (IS). Zooming in on the electropherogram reveals the separation of the different RNA states at 0,300 and $600 \mathrm{nM}$. Clear evidence shows that the fluorescence ratio of the bound RNA over IS increases with higher concentrations of $\mathrm{CDG}$, and quantification shows good linearity. In summary, the 96-plex $f s$ PAG is a robust high-throughput platform for EMSA and possesses great quantitative precision.

\section{CONCLUSION}

We have successfully integrated $f_{S} \mathrm{PAG}$ and EMSAs to form a massive and robust molecular binding reaction screening platform. Careful optimization of the platform enabled low-dispersion injections and improved electrophoresis stability with minimized gel evaporation. Concern of non-uniformity of 96-plex separation was addressed by a geometric redesign. 96-plex EMSAs for CDG riboswitch binding reactions were demonstrated with good linearity over a titration.

Overall, the low-infrastructure yet powerful $f s$ PAG EMSAs offers a promising platform for rapid and reliable binding reaction analysis.

\section{ACKNOWLEDGEMENT}

The authors acknowledge the Herr Lab members for helpful discussion. This work was supported by NSF C AREER Award (CBET-1056035 to A.E.H.). A.E.H. is an Alfred P. Sloan Foundation Research Fellow in chemistry.

\section{REFERENCE}

1. T.A. Duncombe, A.E. Herr, "Photopatterned free-standing polyacrylamide gels for microfluidic protein electrophoresis", Lab Chip, 13 (11), 2115-2123 (2013).

2. J.C. Owicki, "Fluorescence polarization and anisotropy in high throughput screening: Perspectives and primer", J Biomol Screen, 5 (5), 297-306 (2000).

3. L. Mere, T. Bennett, P. Coassin, P. England, B. Hamman, T. Rink, S. Zimmerman, P. Negulescu, "Miniaturized FRET assays and microfluidics: key components for ultra-high-throughput screening”, Drug Discov Today, 4 (8), 363-369 (1999).

4. J.W. Choi, D.K. Kang, H.Park, A.J. deMello, S.I. Chang, "High-throughput analysis of protein-protein interactions in picoliter-volume droplets using fluorescence polarization", Analytical chemistry, 84 (8), 3849-3854 (2012).

5. M. Srisa-Art, D.K. Kang, J. Hong, H. Park, R.J. Leatherbarrow, J.B. Edel, S.I. Chang, A.J. deMello, "Analysis of Protein-Protein Interactions by Using Droplet-Based Microfluidics", Chembiochem, 10 (10), 1605-1611 (2009).

6. C.A. Emrich, H.J. Tian, I.L. Medintz, R.A. Mathies, "Microfabricated 384-lane capillary array electrophoresis bioanalyzer for ultrahigh-throughput genetic analysis", Analytical chemistry, 74 (19), 5076-5083 (2002).

7. D. Ross, J.G. Kralj, "Simple Device for Multiplexed Electrophoretic Separations Using Gradient Elution Moving Boundary Electrophoresis with Channel Current Detection", Analytical chemistry, 80 (24), 9467-9474 (2008).

8. K. Karns, J.M. Vogan, Q. Qin, S.F. Hickey, S.C. Wilson, M.C. Hammond, A.E. Herr, "Microfluidic Screening of Electrophoretic Mobility Shifts Elucidates Riboswitch Binding Function", Journal of the American Chemical Society, 135 (8), 3136-3143 (2013).

9. K.F. Blount and R.R. Breaker, "Riboswitches as antibacterial drug targets", Nat. Biotechnol., 24, 1558-1564 (2006).

\section{CONTACT}

*A.E. Herr, tel: +1-510-666-3396; aeh@berkeley.edu 